\title{
A System for the Acquisition and Analysis of Three-Dimensional Data Describing Dental Morphology
}

\author{
Pingzhou Liu, Sarbin Ranjitkar, John A. Kaidonis, Grant C. Townsend, and Lindsay C. Richards* \\ Dental School, Faculty of Health Sciences, The University of Adelaide
}

ABSTRACT: Accurate, reproducible three-dimensional (3D) data provide an important contribution to our ability to describe, compare and understand dental morphology but the existing technology is often expensive or has technical limitations. Recently available, inexpensive 3D profilometers interfaced with standard personal computers offer the potential to overcome some of these problems. This technical note describes a system that uses a 3D profilometer and purpose written software to analyse changes in dental

A number of systems for the acquisition and analysis of three-dimensional (3D) data have been developed (Roylet et al., 1983; Delong et al., 1985; McDowell et al., 1988; Hewlett et al., 1992) specifically for use in studies of dental morphology. In most cases the developers have recognised difficulties including hardware limitations, computational complexity or cost. In studies of dental morphology the practical problems are usually associated with the complex morphology of teeth that can make it impossible to scan parts of a surface from some directions or lead to with difficulties in defining appropriate reference points and planes. In some cases "internal" reference points (i.e., anatomical features or prepared reference markers) are defined and scanning can extend either to a limit defined by another reference point or a predetermined distance. Alternatively, in some studies appropriate "external" reference points (eg points on the specimen mount) can be defined.

This paper describes the application of widely available hardware and software packages to provide an affordable system for acquiring 3D coordinates from the surface of a dental crown and subsequently for comparing three-dimensional data derived from these coordinates. As part of the development process, the system was validated by comparison of calculated data with the known dimensions and volumes of standard objects. The reproducibility of the derived data, both within and between observers, was also determined from repeated measurements.

To illustrate the application of the system, we have measured the loss of tooth occurring during simulated tooth wear. However, the software and hardware have morphology resulting from tooth wear. The validity of the derived data was determined by comparing data derived from scans of objects of known dimensions with calculated volumes. These differences were less than $10 \%$ from objects that were difficult to scan because of their geometry and were commonly less than $5 \%$. The reproductibility, expressed as intra- and inter-observer coefficients of variation, was less than $1 \%$. The potential applications of systems of this type are outlined. Dental Anthropology 2004;17(3):70-74.

the flexibility to provide valid, reproducible data in a broad range of studies of morphology.

\section{MATERIALS AND METHODS}

For data acquisition a 3D scanner (PIX-4, Roland DG, Tokyo, Japan) interfaced with a personal computer was used to record the heights $(Z)$ of surface mesh points ( $\mathrm{X}$ and $\mathrm{Y}$ ). In this system an active piezo sensor detects contact between its stylus and the scanned surface (Fig. 1). The $X$ and $Y$ mesh steps can be set between $50 \mu$ and $5.00 \mathrm{~mm}$ in $50 \mu$ steps and the Z-axis direction has a resolution of $25 \mu$. The "Dr.PICZA" software (Roland DG, Tokyo, Japan) provided with the scanner is a Windows or MAC OSX-based tool that allows the scan area to be defined to accommodate the dimensions of the specimen and the scanning resolution to be set according to the user's needs. This decision involves balancing the need for high resolution against the size of the resultant data set and the scanning duration, both of which are increased with increasing resolution. In addition, a lower limit and the approximate $\mathrm{X}$ and $\mathrm{Y}$ coordinates of the highest point of the specimen can be defined to further optimise the size of the data set and shorten the scanner's calibration and scanning times. The software allows basic manipulation and visualization of the data (Fig. 2) and has the facility to export data in a range of

\footnotetext{
*Correspondence to: Lindsay Richards, Dental School, Faculty of Health Sciences, The University of Adelaide, Adelaide 5005, South Australia

E-mail: lindsay.richards@adelaide.edu.au
} 


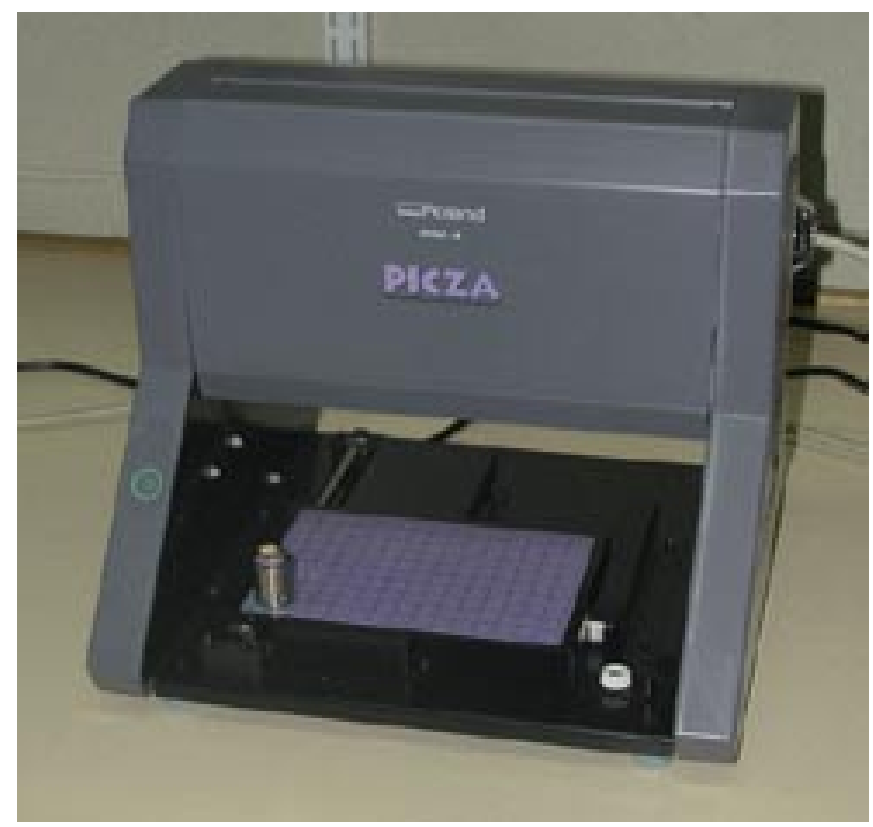

Fig. 1. Scanner with specimen mounted on scanning table.

formats for subsequent analysis.

In our example, we were aiming to measure the changes in dental crown volume resulting from simulated tooth wear. We therefore mounted our specimens (in this case either the buccal of lingual halves of human tooth crowns) with three reference markers (2 $\mathrm{mm}$ diameter titanium spheres) equally spaced around the specimen. After each period of simulated wear the specimen was re-scanned and the volume of the crown above the reference plane compared with previous volumes. Because the predicted changes were relatively small (expected to be of the order of $20 \mathrm{~mm}^{3}$ ) we chose the highest scanning resolution (i.e., $50 \mu$ for the $X$ and $Y$ matrix and $25 \mu$ for the height $(Z))$. The derived data set was exported as a text file for detailed analysis.

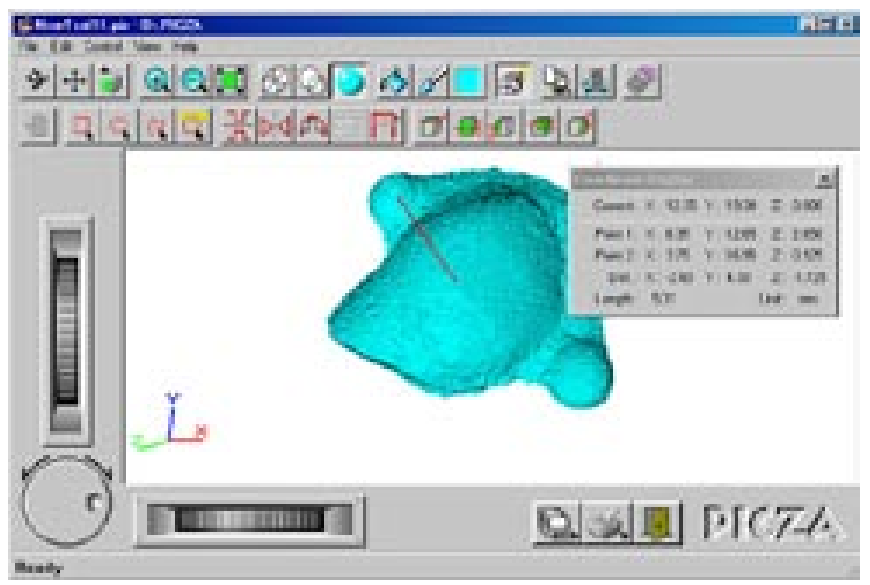

Fig. 2. Data visualization and co-ordinate display from "Dr Picza" software.

For data analysis, a purpose-written software package was developed using MATLAB (version 6, The Mathworks Inc, Natick MA, U.S.A.). The package accepts data from "Dr PICZA" in the form of (X, Y, Z) triples, where the $X$ values are the west-east coordinates and the $Y$ values the north-south coordinates. To make optimum use of MATLAB and its graphic facilities, we converted the data set to a regular mesh grid and saved the $Z$-values to a matrix $(\mathrm{Z})$. The menu-driven software package then provides a series of options for defining the reference plane, graphing the data in $3 \mathrm{D}$ and deriving data describing the volume of the scanned object and the surface area and the height of the highest point on the object from the reference plane in cases where this is of interest.

In our example, we needed to find the volume bounded by two surfaces: the tooth surface and a planar surface defined by the three external reference points. The data transferred from Dr PICZA were plotted using the MATLAB routines and the maximum heights of

Table 1. Three-way analysis of variance comparing 10 repeated measures from two scans of a single specimen performed by three independent observers

\begin{tabular}{lccccc}
\hline \multicolumn{1}{c}{ Source } & Sum Squares & d.f. & Mean Square & F-Ratio & P-Value \\
\hline Repeated measures & 8.7303 & 9 & 0.97003 & 37.89 & $<0.001$ \\
Observers & 0.0287 & 2 & 0.01437 & 0.56 & 0.5803 \\
Scans & 0.0003 & 1 & 0.00026 & 0.01 & 0.9214 \\
Repeat-x-Observer & 0.8173 & 18 & 0.04541 & 1.77 & 0.1169 \\
Repeat-x-Scan & 0.1028 & 9 & 0.01143 & 0.45 & 0.8914 \\
Observer-x-Scan & 0.1011 & 2 & 0.05054 & 1.97 & 0.1678 \\
Error & 0.4608 & 18 & 0.02560 & & \\
Total & 10.2414 & 59 & & & \\
\hline
\end{tabular}




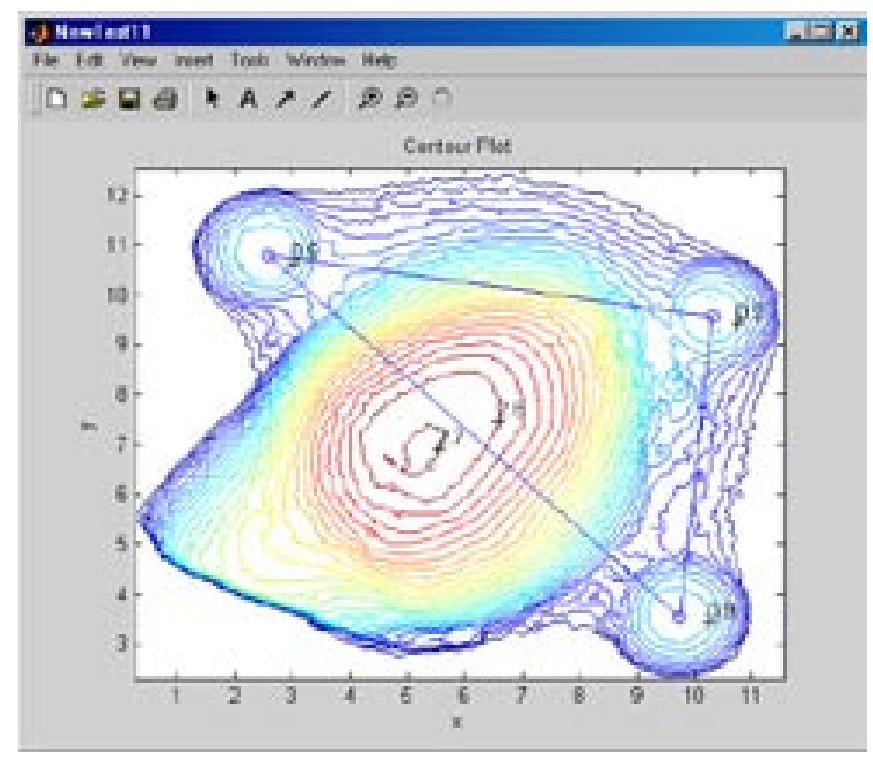

Fig. 3. Data plot and reference plane identification using purpose-written software. the three reference points were identified (Fig. 3). The volume of the part of the specimen above the reference plane was calculated.

Because 3D objects can on occasions include undercut areas, the package has an option to allow the reference plane to the re-aligned so that the calculations do not include parts of the specimen for which there are no data.

In our example this was undertaken by selecting the appropriate option from the menu, inspecting the graphical display and deciding on an appropriate realignment to avoid the undercut and recalculating the data (Fig. 4).

To establish the validity of the data obtained, objects of know dimensions were scanned and the calculated volumes compared with the volumes derived from the scanner data.

To establish the reproducibility of the method, intraobserver variation was assessed by repeated analysis by one observer (PZ) and inter-observer variation was determined by comparing data derived by different

Does the object have undercut areas $(\mathrm{Y} / \mathrm{N})$ ? y

Please give lift value of the referenceplane, which you can estimate from the contour diagram you have drawn.

Lift value $=1.012$

Max distance of the remnant to reference plane is: $0.7130 \mathrm{~mm}$

The convex surface area of the remnant is: $2.4011 \mathrm{~mm}^{\wedge} 2$

The volume of the remnant is: $7.6169 \mathrm{~mm}^{\wedge} 3$

If you want to see the 3-D picture of the original object and reference plane together, please press ' $Y$ ', otherwise just press $<$ Enter $>$.

Selection $(\mathrm{Y} / \mathrm{N}): \mathrm{y}$

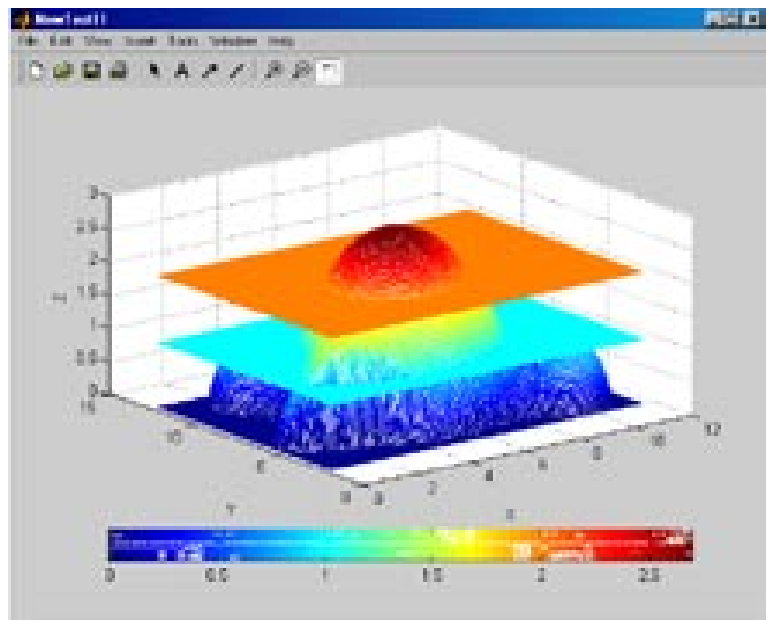

Congratulations! Your measurement of NewTest11 is done.

Matrix: Distance*, Surface*, Volume

$B=\quad 0.7130 \quad 2.4011 \quad 7.6169$

Fig. 4. Example of menu-driven adjustment to reference plane height to avoid undercut areas on specimen. 

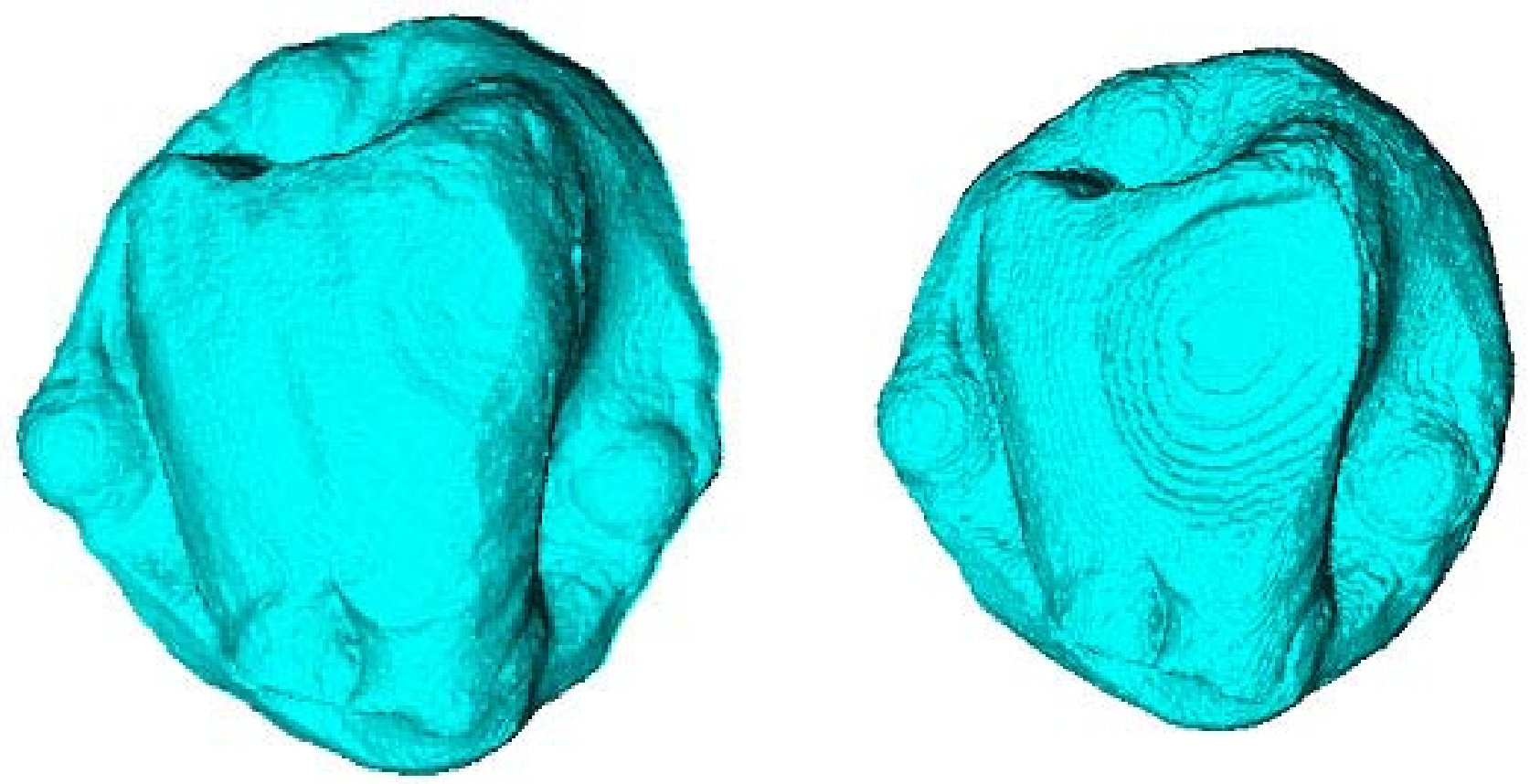

Fig. 5. (left) Data plot for specimen after 7,000 cycles of wear. (right) Data plot for specimen after 112,000 cycles of wear.

observers.

\section{RESULTS}

Scanning a relatively simple object (for example, a hemisphere of diameter $18.0 \mathrm{~mm}$ ) gave a volume of $1583.27 \mathrm{~mm}^{3}$ compared with a calculated volume of $1526.81 \mathrm{~mm}^{3}$. The difference $\left(56.45 \mathrm{~mm}^{3}\right)$ represents $3.7 \%$ of the true volume. A smaller, more complex object (the small cylindrical projection on a Lego ${ }^{\circledast}$ building block which has parallel sides and hence can have small undercut areas if the block is not mounted exactly horizontally) had a theoretical volume of $32.66 \mathrm{~mm}^{3}$ and a volume derived from the scanning data of $35.45 \mathrm{~mm}^{3}$ representing a difference of $8.5 \%$.

The intra-observer variation in calculated volume was small with the coefficient of variation ( $100 \times$ standard deviation/mean) being $0.90 \%$ for the most experienced observer and $0.91 \%$ for the least experienced observer.

To determine whether inter-observer variation or differences between repeated scans of the same object contributed significantly to the observed variation in repeated measures, one specimen was scanned on two occasions and each of these scans was analysed 10 times by three independent observers. A three-way analysis of variance (Table 1) revealed no significant variation between observers $(p=0.58)$ or between repeated scans $(\mathrm{P}=0.92)$ and no significant interaction between any of the considered factors suggesting that the performance of experienced and inexperienced observers was similar.

In our study of tooth wear the buccal surface of an extracted human tooth was subjected to 7000 cycles at the rate of 80 cycles per minute under a load of $3.2 \mathrm{~kg}$ with water at $\mathrm{pH} 7$ used as a lubricant in an electromechanical tooth wear machine (Kaidonis et al., 1998) to produce a wear facet (Fig. 5a). The specimen was scanned and the volume of the dental crown above the plane defined by the three $2 \mathrm{~mm}$ diameter ball markers that were used as "external" reference points was calculated. This was compared with volume of the specimen after it had been subjected to a further 105,000 cycles of wear (Fig. 5b). The volume of enamel lost due to wear during this experiment $\left(21.85 \mathrm{~mm}^{3}\right)$ was calculated by comparing the first volume $\left(149.74 \mathrm{~mm}^{3}\right)$ with the final volume $\left(127.89 \mathrm{~mm}^{3}\right)$.

\section{DISCUSSION}

Based on our assessment of the validity and reproducibility of the measurements derived using this system, we believe that it provides an affordable and reliable method for the acquisition of $3 \mathrm{D}$ data for the comparison of dental morphology. Like most systems it is limited in its ability to deal with undercut areas that makes it important to carefully select the initial orientation of the specimen and define an appropriate reference plane to avoid undercuts. Also, the acquisition of data from larger specimens at the highest resolution can be time consuming with high resolution scanning of a whole dental arch taking up to 30 or more hours.

The costs involved in setting up the system are relatively small compared with some other systems. If a suitable personal computer and a licensed copy of the MATLAB package are available then the total hardware 
set up cost should be less than $\$ U S$ 1,200 compared with more than \$US 100,000 for some commercial laser-based systems. The purpose-written MATLAB-based software package is available on request from the authors.

The validity of the data derived using the system was established by comparing volumes derived by scanning with the calculated volumes of objects of known dimensions. This indicated that calculated and scan-derived volumes differed by between 3.7 percent and 8.5 percent depending on the size and geometry of the specimen. Interpreting this information was complex for a number of reasons. In the case of the sphere (a computer-mouse ball), the difference between the scanned and calculated volumes was relatively small and challenged our ability to accurately measure the ball. A difference in radius of the ball of the order of 0.1 $\mathrm{mm}$ would result in a difference in volume of more than $3.4 \%$ and made it difficult to determine which of the calculated and scan-derived data was the more valid. In the case of the Lego ${ }^{\circledR}$ building block the differences were larger because the object presented some obvious and some hidden challenges and represented a "worst case" in terms of the ability of the system to derive valid data. The obvious challenge was the geometry of the object that, with its parallel sides, required precise orientation to avoid undercuts. In addition, the curvefitting procedures that were used to define the surface were not ideally suited to objects of this type. The hidden challenge was the surface morphology of the object. The face of the projection on the block included the manufacturer's trademark etched into the surface. This was not obvious on observation and therefore not included in the calculations but was obvious on the enlarged scan and would contribute to the difference between the calculated and scan-derived volumes.

The reproducibility of the data was assessed by repeated measures of a test specimen by different observers. The intra- and inter-observer errors were all small with coefficients of variation for repeated measures being less than $1.0 \%$ for all scans and observers, and with no significant differences between observers or repeated scans.

Based on our experience, we believe that the system described is an affordable, valid and reliable method for obtaining 3D data for the description and comparison of dental morphology.

\section{ACKNOWLEDGMENTS}

The support of the National Health and Medical Research Council of Australia and the assistance of Dr Shosei Eguchi, Nihon University School of Dentistry at Matsudo, Japan and Heather Lewis are acknowledged.

\section{LITERATURE CITED}

Delong R, Pintado M, Douglas WH. 1985. Measurement of change in surface contour by computer graphics. Dental Materials 1:27-30

Hewlett ER, Orro ME, Clark GT. 1992. Accuracy testing of three dimensional digitizing systems. Dental Materials 8:49-53

Kaidonis JA, Townsend GC, Richards LC, Tansley GD. 1998. Wear of human enamel: a quantitative in vitro assessment. J Dent Res 77:1983-1990

McDowell GC, Bloem TJ, Lang BR, Asgar K. 1988. In vivo wear. Part 1: The Michigan computer graphic measuring system. J Prosthet Dent 60:112-120

Roylet JF, Reich T, Lutz F. 1983. High precision occlusal mapping: a new method for measuring wear of posterior composites. J Dent Res 62:220 (Abstract). 